\section{P063 THE USE OF TRANSJUGULAR INTRAHEPATIC PORTOSYSTEMIC SHUNTS (TIPS) IN TREATMENT OF ASCITES IN HEPATIC SYSTEMIC MASTOCYTOSIS}

Hamish Miller*, David Patch, r Dominic Yu. Royal Free Hospital, London, UK

\subsection{6/gutjnl-2021-BASL.72}

Introduction Systemic mastocytosis is a rare cause of portal hypertension which can lead to the development of ascites. ${ }^{1-}$ Transjugular intrahepatic portosystemic shunts (TIPS) can be used as a management option for refractory ascites. ${ }^{2}$ We describe a tertiary experience of 2 cases of hepatic systemic mastocytosis complicated by ascites which have improved following insertion of a TIPS. There is optimism regarding the use of KIT inhibitors such as avapritinib in systemic mastocytosis which my improve outcomes and therefore patients with hepatic involvement may survive longer and could be affected by complications of portal hypertension such as ascites. ${ }^{3}$ It is therefore important to explore management options in these patients.

Discussion The first patient is a 71 year old female with systemic mastocytosis who developed ascites in December 2020 which required paracentesis every 3 weeks. She subsequently had a liver biopsy which was consistent with systemic mastocytosis. The liver biopsy showed a mast cell infiltrate centred on portal tracts but extending along sinusoids to central veins. Mast cells are positive for CD117 and CD25 but negative for MCT. She was worked up for a TIPS insertion which occurred in March 2021. Prior to TIPS, the pressures in the portal vein and inferior vena cava (IVC) were $25 \mathrm{mmHg}$ and $10 \mathrm{mmHg}$ respectively. Post TIPS, the pressure in the portal vein was $18 \mathrm{mmHg}$ and the pressure in the IVC was $13 \mathrm{mmHg}$. Her ascites improved in the following months but did not completely resolve.

The second patient is a 69 year old male who initially had a low risk myelodysplastic syndrome who subsequently developed systemic mastocytosis which was confirmed on bone marrow biopsy. He developed ascites in September 2020 requiring ascitic paracentesis. He had a TIPS placed in November 2020. His portal vein pressure prior to stenting was $24 \mathrm{mmHg}$ and $18 \mathrm{mmHg}$ afterwards. By February 2021, there had been complete resolution of his ascites.

Conclusion We conclude that a TIPS is a good management option for refractory ascites in this cohort of patients but recognise that this is a small sample size and needs to be further investigated. To the best of our knowledge, there are no cases published on the use of TIPS for ascites in hepatic systemic mastocytosis and therefore these cases give a unique insight into this management option.

\section{REFERENCES}

1. Martins $C$, et al. Systemic mastocytosis: a rare cause of non-cirrhotic portal hypertension. World J Gastroenterol 2016;22:6559-6564.

2. Adebayo D, Neong SF, Wong F. Refractory ascites in liver cirrhosis. Am J Gastroenterol 2019;114:40-47.

3. Gilreath JA, Tchertanov L, Deininger MW. Novel approaches to treating advanced systemic mastocytosis. Clin Pharmacol Adv Appl 2019;11:77-92.

\section{P064 A CLOSE LOOP AUDIT OF HEPATOCELLULAR CARCINOMA SURVEILLANCE: IMPACT OF COVID PANDEMIC}

Rudrik Thakkar*, Aqeel Jamil. Salisbury NHS Foundation Trust, Salisbury, UK
Introduction Cirrhosis is a significant risk factor for hepatocellular carcinoma (HCC). HCC develops rapidly and asymptomatic until advanced stage. ${ }^{1}$ The incidence of HCC in patients with cirrhosis is $2.5 \%$ per year. ${ }^{2}$ NICE guidelines recommend offering HCC surveillance to patients with cirrhosis using ultrasound scan (US) and serum alpha fetoprotein (AFP) every six months. ${ }^{1}$ We undertook a close loop audit to assess performance of HCC surveillance at the local hospital and assessed the impact of the COVID pandemic on the service.

Method Data was collected on patients admitted to hospital with cirrhosis in 1 st cycle (16/12/18-05/04/19) and 2 nd cycle (4/12/19-31/05/20). Intervention was performed on 04/12/ 2020 which included departmental posters and informing the doctors on Gastroenterology ward of the NICE guidelines. Data was collected on US and AFP follow up six months after the discharge. Exclusion criteria were patients under the age of 18, patients with known metastasis and on palliative management.

Results Total number of patients were 55 and 42 in the 1 st and 2 nd cycle respectively. Average age was 65 in the 1 st cycle and 61 in the 2 nd cycle. Overall, $48.5 \%$ were male and $51.5 \%$ were female patients. 6 monthly ultrasound follow up in patients reduced from $30.9 \%$ in 1 st cycle to $23.8 \%$ in 2 nd cycle during the COVID pandemic. Similarly, 6 monthly AFP follow-ups reduced from $34.5 \%$ in 1 st cycle to $23.8 \%$ in 2 nd cycle during the COVID pandemic. Although US and AFP follow-ups organised by the gastroenterology ward on discharge were $50 \%$ of all outpatients in six months in the 2 nd cycle.

Discussion COVID Pandemic, with the 1st UK lockdown taking place on 23/03/2020, has had a significant impact on the HCC surveillance of patients who were discharged from hospital. This could be due to disruption to the service provided by the hospital or the patients' choice to avoid attending hospital during the COVID pandemic. The audit demonstrated that despite the disruption to the overall surveillance, the change implemented has resulted in people being followed up for HCC surveillance than previously when no follow up was organised by the discharging team at the hospital as this is mostly managed by the outpatient nurses. A re-audit following COVID pandemic would be useful to understand any benefit of such inpatient intervention on outpatient follow-up of patients with cirrhosis.

\section{REFERENCES}

1. Quality statement 4: Surveillance for hepatocellular carcinoma | Liver disease Quality standards | NICE [Internet]. Nice.org.uk. 2021 [cited 28 June 2021]. Available from: https://www.nice.org.uk/guidance/qs152/chapter/Quality-statement-4 Surveillance-for-hepatocellular-carcinoma\#quality-statement-4

2. Mancebo A, González-Diéguez $M$, Cadahía V, Varela $M$, Pérez $R$, Navascués $C$ et al. Annual incidence of hepatocellular carcinoma among patients with alcoholic cirrhosis and identification of risk groups. Clinical Gastroenterology and Hepatology 2013;11(1):95-101.

\section{P065 GENETIC EVALUATION OF PATIENTS LISTED FOR LIVER TRANSPLANTATION WITH CRYPTOGENIC CIRRHOSIS}

${ }^{1}$ Jeremy Nayagam*, ${ }^{2}$ Rhiannon Taylor, ${ }^{3}$ Elizabeth Wlodek, ${ }^{4}$ Melanie Cuffe, ${ }^{5}$ Neil Halliday, ${ }^{6}$ Steven Masson, ${ }^{4}$ Vinod Hegade, ${ }^{5}$ Jonathan Potts, ${ }^{7}$ Anya Adair, ${ }^{7}$ Timothy Gordon-Walker, ${ }^{8}$ James Ferguson, ${ }^{3}$ Bill Griffiths, 'Deepak Joshi. ${ }^{1}$ King's College Hospital, London, UK; ${ }^{2} N H S$ Blood and Transplant, Bristol, UK; ${ }^{3}$ Addenbrooke's Hospital, Cambridge, UK; ${ }^{4}$ St James' University Hospital, Leeds, UK; ${ }^{5}$ Royal Free Hospital, London, UK; ${ }^{6}$ Freeman Hospital, Newcastle, UK; ${ }^{7}$ Royal Infirmary of Edinburgh, Edinburgh, UK; ${ }^{8}$ Queen Elizabeth Hospital, Birmingham, UK 
Introduction Identifying the underlying cause of liver disease is important, however in 5-30\% of patients with cirrhosis no cause can be found, which is referred to as cryptogenic cirrhosis (CC). From registry data $4 \%$ of liver transplants are performed for CC. UK Transplant Registry data suggests CC recipients have a lower 1 -year and 5-year survival than the national rate.

Understanding of the genetics underpinning liver disease has evolved significantly. More recently whole exome sequencing (WES) has been proposed for the evaluation of cryptogenic liver disease.

We sought to evaluate the diagnostic work-up of patients listed for transplant in the UK with CC, with a specific focus on the use of genomics.

Method A retrospective search of the UK Transplant Registry was performed for adult elective NHS patients registered for first liver transplant at 6 transplant centres between 1/1/15 and $31 / 12 / 20$. Patients with CC or genetic liver disease as an indication at registration were included. Clinical information and results of diagnostic tests were collected.

Results After exclusion of 59 patients (42 genetic co-factor, 17 alternate aetiology), 228 patients were included: 120 CC, 4 diagnosed with genetic liver disease during assessment, 104 known genetic liver disease.

The patients without previously known genetic liver disease $(n=124)$ had a median age at onset of liver disease of 49 years (IQR, 37-56), 82 (66\%) were male, 16 (13\%) had a family history of liver disease. Median age at listing was 55 years (IQR, 42-62), UKELD 56 (52-59) and MELD 15 (1220). Prior diagnostics included: liver biopsy 80 (65\%), cross sectional imaging 123 (99\%), and cholangiography 26 (21\%).

A genetic test was performed in $29(23 \%)$ patients: 10 ATP7B, $20 \mathrm{HFE}, 9$ cholestasis gene panel, and 1 underwent WES. There were 4 new diagnoses of genetic liver disease made during evaluation: 2 MDR3 deficiency, 1 Wilson disease, 1 alpha-1-antitrypsin. In 2 other patients there were ABCB4 variants of uncertain significance and potential MDR3 deficiency.

Discussion Genetic sequencing is performed in selected patients assessed for transplant with CC, and in some cases a new diagnosis of genetic liver disease has been made. With increasing access to genetic testing and the NHS Long Term Plan to offer whole genome sequence as routine care, more widespread use of genetic testing in patients with CC listed for transplant should be considered.

\section{P066 DNA METHYLATION DIFFERENCES AND METHYLATION QUANTITATIVE TRAIT LOCI IN PRIMARY SCLEROSING CHOLANGITIS AND IGG4-RELATED SCLEROSING CHOLANGITIS}

Alex Adams*, Silvia Cabras, Belén Morón-Flores, Alessandra Geremia, Emma Culver, Jack Satsangi. University of Oxford, UK

\subsection{6/gutjnl-2021-BASL.75}

Primary sclerosing cholangitis (PSC) and IgG4-related sclerosing cholangitis (IgG4-SC) are fibro-inflammatory conditions of the liver and biliary tree, driven by a complex interplay of genetic, environmental, and immunologic factors. Epigenetic studies sit at the interface between genes and the environment. In previous work we have defined replicable alterations in new-onset adult (Ventham et al, Nat Commun 2016;7:13507) and paediatric inflammatory bowel disease (Adams et al, Inflamm Bowel Dis 2014;20:1784). We have now assayed methylomes of patients with PSC and IgG4-SC to ascertain epigenetic signatures that may provide insight into pathogenesis and novel biomarkers, and compared these with the methylome of ulcerative colitis (UC) and healthy controls (HC).

DNA methylation profiling in whole blood and genotyping was performed in 70 PSC, 56 IgG4-SC, $66 \mathrm{UC}$, and $88 \mathrm{HC}$ samples, with Infinium MethylationEPIC and Global Screening arrays (Illumina). UC patients were selected to match the clinical characteristics (extent, activity) of the PSC-UC cohort. Analysis was performed with R (v4.0.3). DNA methylation data was processed with Minfi, batch effects were treated with ComBat and associations were tested using limma. Genotyping was analysed with Plink (v1.9) and meQTLs with linear models in R. Statistical significance was determined using the Holm method.

Compared to HC, 31 probes showed significant methylation differences in PSC, and 13 in IgG4-SC, with 8 probes shared between conditions. In separate subanalyses of PSC-UC and PSC without IBD $(n=13)$ we found 25 significant probes

Abstract P065 Table 1 Demographics, clinical characteristics and biochemistry of study population at time of listing for transplant

\begin{tabular}{|c|c|c|c|c|c|c|}
\hline & Cryptogenic cirrhosis & Alpha-1-antitrypsin & Haemochromatosis & Cystic fibrosis & Wilson disease & MDR3 deficiency \\
\hline Number & 120 & 42 & 41 & 10 & 9 & 5 \\
\hline Age at listing median (IQR), years & $56(44-63)$ & $54(49-63)$ & $61(58-68)$ & $28(23-37)$ & $31(25-36)$ & $31(27-40)$ \\
\hline Male $\%$ & $78(65 \%)$ & $32(76 \%)$ & $36(88 \%)$ & $6(60 \%)$ & $6(67 \%)$ & $4(80 \%)$ \\
\hline BMI median (IQR), years & $27.5(23.9-30.9)$ & $30.0(25.1-35.8)$ & $28.2(25.9-31.6)$ & $19.8(19.2-20.7)$ & $22.2(21.2-28.9)$ & $23.4(22.3-24.8)$ \\
\hline $\mathrm{HCC} \%$ & $17(14 \%)$ & $5(12 \%)$ & $26(63 \%)$ & 0 & 0 & 0 \\
\hline MELD median (IQR) & $15(12-20)$ & $15(12-19)$ & $11(7-14)$ & $12(10-14)$ & $21(14-28)$ & $21(17-25)$ \\
\hline UKELD median (IQR) & $56(52-59)$ & $55(52-57)$ & $51(48-55)$ & $54(50-55)$ & $56(53-66)$ & $64(58-66)$ \\
\hline ALT median (IQR), IU/L & $41(24-57)$ & $41(29-54)$ & $32(24-39)$ & $35(14-59)$ & $84(42-408)$ & 142 \\
\hline AST median (IQR), IU/L & $63(47-85)$ & $71(56-99)$ & $63(39-78)$ & $39(32-42)$ & $88(60-133)$ & $238(113-369)$ \\
\hline ALP median (IQR), IU/L & $154(116-241)$ & $144(107-177)$ & 115 (89-154) & $190(90-385)$ & 107 (90-149) & $303(189-618)$ \\
\hline GGT median (IQR), U/L & $84(41-144)$ & $68(36-129)$ & $72(40-111)$ & $80(36-339)$ & $112(36-197)$ & $151(68-211)$ \\
\hline
\end{tabular}

